\title{
Pigmented Lesions of Ocular Fundus: Clinical Aspects
}

\author{
Maria Antonietta Blasi ${ }^{1}$, Maria Grazia Sammarco ${ }^{1}$, Andrea Scupola ${ }^{1}$, Martina Maceroni*2, \\ Giulia Midena*2 and Monica Maria Pagliara ${ }^{1}$ \\ ${ }^{1}$ Dipartimento Testa Collo, Italia \\ ${ }^{2}$ Istituto di Oftalmologia, Italia \\ *Corresponding author: Martina Maceroni, Istituto di Oftalmologia, Italia \\ Giulia Midena, Istituto di Oftalmologia, Italia
}

\section{ARTICLE INFO}

Received: 画 January 18, 2019

Published: 慧February 01, 2019

Citation: Maria Antonietta B, Maria Grazia S, Andrea S, Martina M, Giulia M, Monica Maria P. Pigmented Lesions of Ocular Fundus: Clinical Aspects. Biomed J Sci \& Tech Res 13(5)-2019. BJSTR. MS.ID.002477.

\begin{abstract}
Several pigmented lesions of the ocular fundus can be detected on routine ophthalmic examination. Pigmented tumoral lesions, both benign and malignant, can arise from the retinal pigment epithelium or from choroidal melanocytes. A careful evaluation of the clinical and imaging features can help to differentiate the lesions, avoiding diagnostic errors.

Keywords: Ocular Tumors; Retinal Pigment Epithelium Lesions; Choroidal Melanocytic Lesions; Choroidal Melanoma; Pseudomelanoma

Abbreviations: RPE: Retinal Pigment Epithelium; CHRPE: Congenital Hypertrophy of the Retinal Pigment Epithelium; FA: Fluorescein Angiography; ICGA: Indocyanine Green Angiography; FAF: Fundus Autofluorescence; US: Ultrasonography; EDI: Enhanced Depth Imaging; OCT: Optical Coherence Tomography; PEHCR: Peripheral Exudative Hemorrhagic Chorioretinopathy
\end{abstract}

\section{Introduction}

Circumscribed pigmented lesions of the ocular fundus are not uncommon. They can be congenital, developmental, neoplastic or the result of infectious or inflammatory processes. Pigmented neoplastic lesions of ocular fundus, both benign and malignant, can arise from the retinal pigment epithelium (RPE) or from choroidal melanocytes. It is important for the ophthalmologist to properly diagnose these lesions to avoid erroneous diagnosis and misdirected treatment.

\section{Discussion}

\section{Lesions Arising from the Retinal Pigment Epithelium}

Congenital hypertrophy of the Retinal Pigment Epithelium (CHRPE) is an uncommon benign fundus lesion. The typical unifocal, unilateral lesion occurs in about $0.5 \%$ of individuals [1]. CHRPE lesions are usually asymptomatic and detected on routine ophthalmic examination. The typical unifocal CHRPE is a gray to black, well-defined, minimally elevated fundus lesion having a diameter in the range of $2-5 \mathrm{~mm}$ (Figure 1 ). The lesion frequently undergoes at least partial depigmentation with aging, developing discrete intralesional atrophic foci (lacunae that tend to enlarge and coalesce over the years). No diagnostic studies are generally indicated for characterization of these retinal lesions. Fluorescein angiography (FA) and indocyanine green angiography (ICGA) show a well-defined blocking defect at the retinal pigment epithelial level. Transmission of choroidal fluorescence takes place through lacunae in the lesion [2]

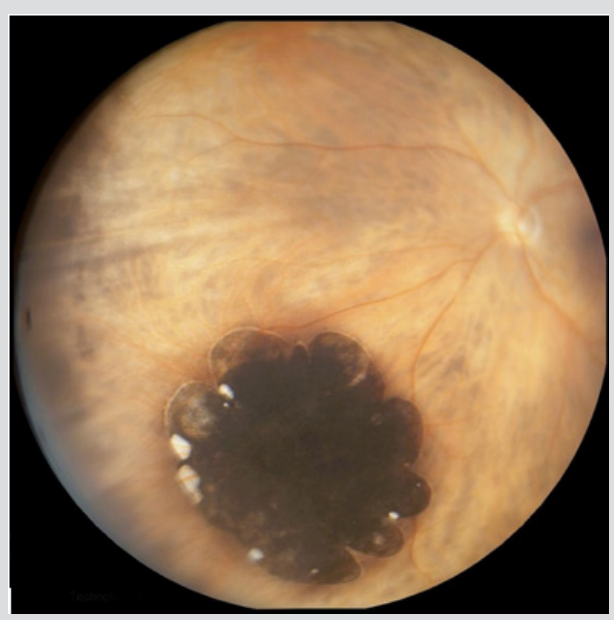

Figure 1: Fundus photography of CHRPE.

The unilateral multifocal CHRPE, also known as congenital grouped pigmentation of the retina or "bear tracks", is characterized by multiple, flat, gray to black RPE clustered in one 
region of the fundus. These lesions are hypofluorescent on FA and hypoautofluorescent on fundus autofluorescence (FAF) [3]. Atypical multifocal bilateral CHRPE presents oval or irregularly shaped lesions, variable in size (usually between 0.1 and $2.0 \mathrm{~mm}$ in maximal diameter), widely separated rather than clustered; it is associated with familial colonic adenomatous polyposis syndrome.1 Reactive Hyperplasia of the Retinal Pigment Epithelium is a result of an ocular insult, such as inflammation or trauma. It can appear as a diffuse or sessile lesion or as a nodular mass. Its dark black color and associated signs of prior inflammation or trauma can help to differentiate the lesion from choroidal melanoma. Major complications or progression are quite unusual [4].

Adenoma and adenocarcinoma of the RPE have respectively benign and malignant histopathologic features and they are generally diagnosed in adulthood, with a mean age of 53 years [5] Unlike uveal melanoma, these tumors seem to have no predilection for race. They may have a slight predilection for females [6] Clinically, these lesions are usually solitary and unilateral and begin as a small, deep retinal tumor that is dark brown to black in color. They usually grow very slowly and invade the overlying sensory retina. In some instances, they have arisen from solitary congenital hypertrophy of the RPE [7] FA can document the retinal feeder vessels and show early hypofluorescence and late minimal hyperfluorescence of the tumor, without visibility of choroidal vessels. Ultrasonography (US) typically demonstrates the tumor to be elevated and to have medium to high internal reflectivity. Enhanced depth imaging optical coherence tomography (EDI-OCT) shows the tumor surface as irregular and dense posterior optical shadowing (Figure 2) [6].

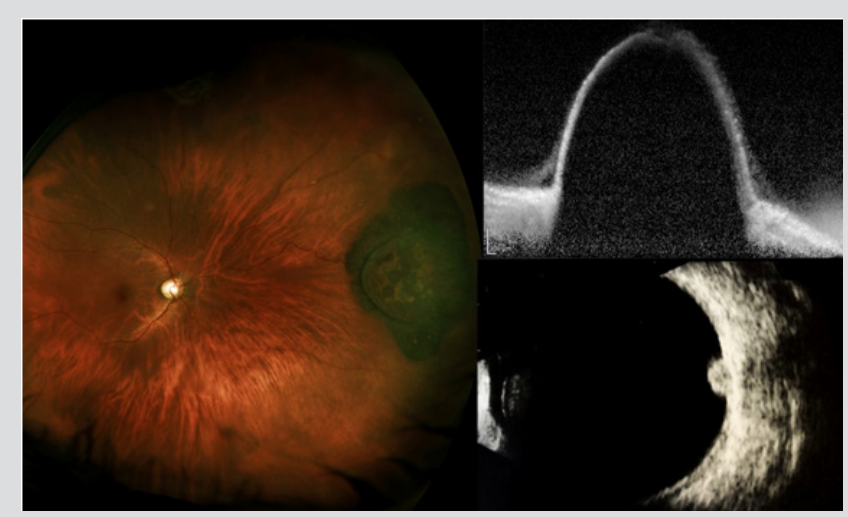

Figure 2: Fundus photography (Left), EDI-OCT (Top Right) and US imaging (Bottom Right) of adenoma of the RPE.

Combined hamartoma of the retina and RPE is a congenital, nonhereditary lesion with an uncertain etiology. This lesion is composed of an admixture of pigment epithelial cells, proliferating blood vessels and glial tissue. It appears as an ill-defined gray retinal mass with tortuous or straightened retinal blood vessels and it is characteristically located on or adjacent to the optic disc, but it can be seen in extrapapillary areas of the fundus. FA shows abnormal retinal blood vessels in the mass and gradual late staining of the lesion. Optical coherence tomography (OCT) shows an irregular lesion with vitreoretinal traction [8].

\section{Lesions Arising from Choroidal Melanocytes}

Choroidal nevus is the most common primary intraocular tumor. In Caucasians, it has been estimated that approximately 2 to $10 \%$ of persons older than 50 years have at least one choroidal nevus [9].It is much more common in persons of lightly pigmented races and it appears to be equally common in men and women. The typical choroidal nevus appears as a small gray to brown tumor. Many choroidal nevi exhibit characteristic surface alterations such as drusen and RPE pigment clumping. Most choroidal nevi are 5 $\mathrm{mm}$ or less in basal diameter and $1 \mathrm{~mm}$ or less in thickness. Blurred or distorted vision attributable to a choroidal nevus may be the result of an accumulation of serous subretinal fluid [10], cystic degeneration of the retina overlying a macular choroidal nevus [11] or choroidal neovascularization [12] Diagnosis of a typical choroidal nevus is based almost exclusively on clinical features; fundus photograph can be useful to compare subsequent follow-up evaluations.

US is commonly used for the measurement of tumor thickness; most choroidal nevi are nearly flat and can be difficult to detect. Choroidal nevus is characterized by high internal reflectivity on US. On FA nevus remains typically hypofluorescent. EDI-OCT can image choroidal nevi, even those undetectable by US: choriocapillaris thinning over the nevus, partial or complete choroidal shadowing deep to the nevus, RPE atrophy, photoreceptor loss and inner segment-outer segment junction irregularity are some of the EDI-OCT features of choroidal nevus (Figure 3). On FAF imaging pigmented nevus generally appears more hypoautofluorescent from the intrinsic melanin that absorbs light compared with less pigmented nevus that appears more hyper-autofluorescent. The estimated annual rate of malignant transformation of a pre-existing nevus into melanoma reported in several studies varied from 0,2 to $1 \%[13]$.

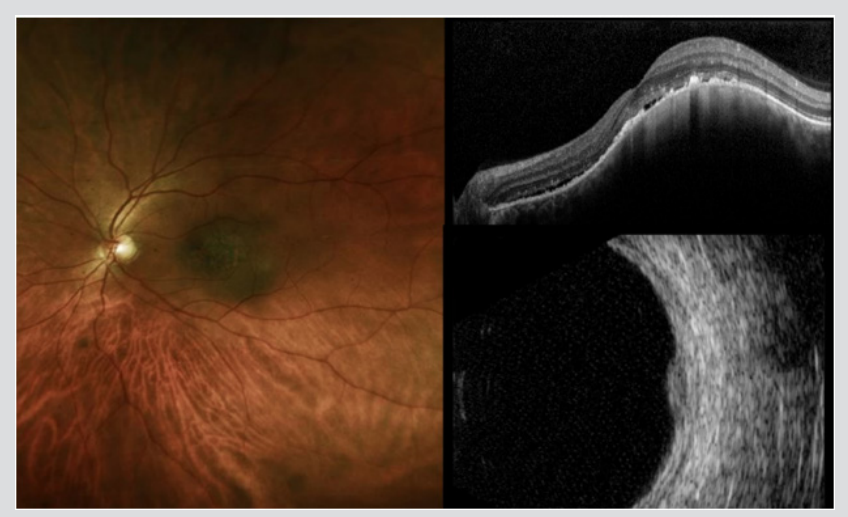

Figure 3: Fundus photography (Left), EDI-OCT (Top Right) and US imaging (Bottom Right) of choroidal nevus.

Aspecial type of uveal nevus is the melanocytoma (magnocellular nevus) of the optic disc. This lesion is usually composed entirely of maximally pigmented, polyhedral nevus cells (magnocellular nevus 
cells). Clinically, the melanocytoma is a dark brown tumor that involves the substance of the optic disc. The surface of the tumor commonly appears striated because of insinuation of the darkly pigmented cells of the tumor between axons in the nerve fiber layer. If the melanocytoma compresses the optic disc, it can produce prominent visual field defects. Lesion enlargement can occur but is usually slow. The diagnosis can be made by ophthalmoscopic recognition of its characteristic clinical features. In most cases, FA of a melanocytoma of the optic disk demonstrates hypofluorescence throughout the angiogram, because the cells are deeply pigmented and closely compact with relatively little vascularity. In cases with optic disk edema, there is hyperfluorescence of the edematous portion of the optic disk. ICGA also shows the lesion to be generally hypofluorescent. US shows high reflectivity of the lesion (Figure 4). Malignant change is estimated to occur in about $1-2 \%$ of cases [14].

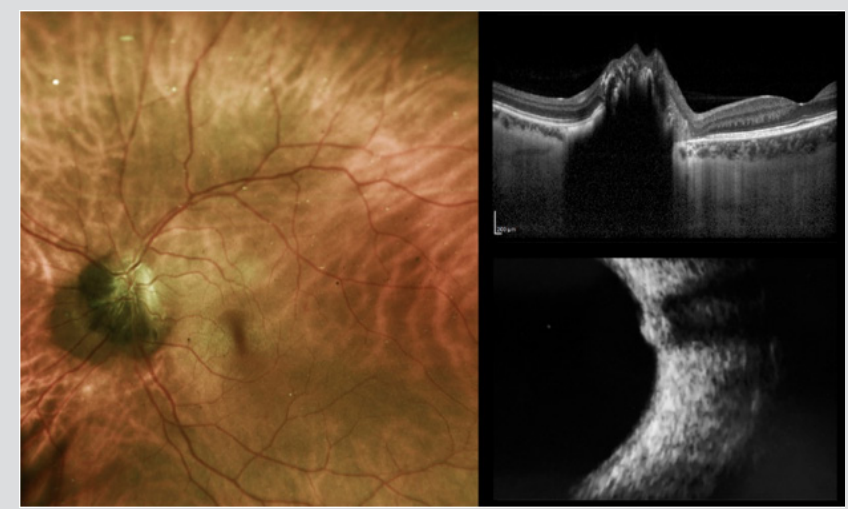

Figure 4: Fundus photography (Left), EDI-OCT and US imaging (Bottom Right) of melanocytoma of the optic nerve.

Choroidal melanoma is the most common primary intraocular malignant tumor, with an incidence of 5.1 cases per million per year [15] Choroidal melanoma can originate from a pre-existing choroidal nevus. In 1995, Shields et al. identified several risk factors predictive of nevus growth into melanoma: thickness more than $2 \mathrm{~mm}$, subretinal fluid, symptoms of flashes/floaters and blurred vision, orange pigment, margin less than or equal to $3 \mathrm{~mm}$ from optic disk, ultrasonographic hollowness and absence of halo . Tumors with three or more risk factors likely represent small melanoma, demonstrate malignant transformation in 50\% of cases at 5 years and treatment should be considered early [16]. The classic appearance of choroidal melanoma is pigmented domeshaped or mushroom-shaped tumor with an associated exudative retinal detachment.

The mushroom-shaped configuration is due to the breaking of the overlying Bruch's membrane and RPE, forming a nodular eruption beneath the retina that progressively enlarge. Although some choroidal melanomas are detected on ophthalmic evaluations prompted by visual symptoms (e.g., blurred vision, visual field defect, flashes, floaters), many patients who have such a tumor are asymptomatic at the time of detection. Small choroidal melanomas can be detected by EDI-OCT and it is also a useful tool for differentiating choroidal nevus from small choroidal melanoma. It has limited use with heavily pigmented tumors, which cast a significant posterior shadow. US is an important diagnostic tool used to define tumor extent and shape, and to measure tumor dimensions of choroidal melanoma. Typical features of a posterior uveal melanoma on US include acoustic hollowing, choroidal excavation and orbital shadowing (Figure 5). Spontaneous vascular movement may be noted in a highly vascularized tumor.

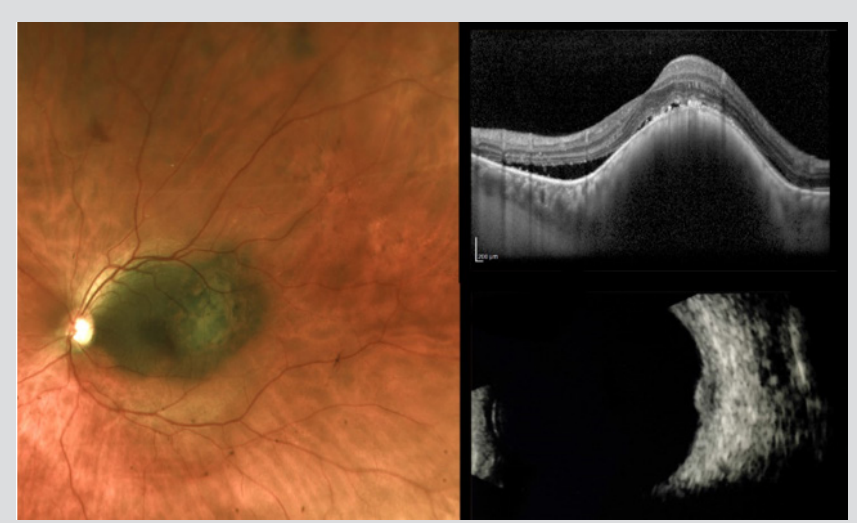

Figure 5: Fundus photography (Left), EDI-OCT and US imaging (Bottom Right) of choroidal melanoma.

On A-scan the four cardinal acoustic hallmarks of malignant melanoma are a regular internal structure with similar height of the inner tumor spikes or regular decrease in height, low to medium reflectivity, solid consistency with no after movement of tumor spikes, and echographic sign of vascularization with a fast, spontaneous, continuous, flickering vertical motion of single tumor spikes. The characteristic features on FA includegradually increasing fluorescence that starts at or before the arterial phase, increases in intensity well into the recirculation phase, and persists for at least $45 \mathrm{~min}$. The tumors that have broken through Bruch's membrane characteristically reveal "double circulation" pattern because of superimposition of the intravascular fluorescence of the intact retinal vasculature over the fluorescence of large caliber vessels within choroidal tumor. ICGA allows better visualization of intrinsic vasculature in choroidal melanoma with maximal fluorescence at an average of $18 \mathrm{~min}$ from injection. Choroidal melanoma generally shows on FAF slight intrinsic hyperautofluorescence and the brightness increases with pigmented tumours, larger tumours, and those associated with disrupted RPE $[17,18]$.

\section{Pseudomelanoma}

There are conditions that can clinically simulate melanoma, leading to diagnostic ambiguity. The most frequent pseudomelanomas included choroidal nevus, peripheral exudative hemorrhagic chorioretinopathy (PEHCR), congenital hypertrophy of the retinal pigment epithelium and idiopathic hemorrhagic detachment of the retina or RPE [18] This section covers selected nonneoplastic-simulating conditions that are not discussed elsewhere in this article. Peripheral exudative hemorrhagic chorioretinopathy (PEHCR) is a peripheral retinal degeneration of the elderly manifesting with exudative and hemorrhagic changes. 
This condition can closely simulate melanoma with a homogenous deep brown mass in the periphery (Figure 6). However, PEHCR shows blockage on FA, dark hypoautofluorescence on FAF, echodensity with retraction cleft on US and spontaneous resolution [19].

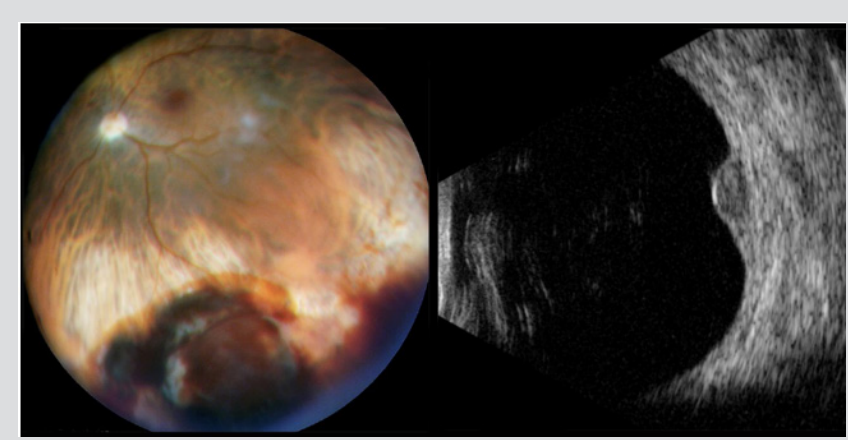

Figure 6: Fundus photography (Left) and US imaging (Right) of PEHCR.

Idiopathic hemorrhagic detachment of the retina occurs after bleeding into the subretinal space and in some cases large amount of blood may accumulate, creating a hemorrhagic detachment of the retina, whereas idiopathic hemorrhagic detachment of the retinal pigment epithelium occurs in a variety of conditions such as senile disciform macular degeneration and angioid streaks, and it may complicate other conditions characterized by breaks in Bruch's membrane. In many of these cases there is a clinically detectable source of the blood, such as an intraretinal microaneurysm [20].

\section{Conclusion}

A variety of pigmented lesions can simulate choroidal melanoma. Suspicious choroidal nevus is still the lesion most difficult to differentiate from choroidal melanoma. Most other pseudomelanomas can lead to diagnostic ambiguity. Some elements can still generate confusion, but careful assessment of the clinical and instrumental characteristics can help to avoid diagnostic errors.

\section{References}

1. Coleman P, Narnard NA (2007) Congenital hypertrophy of the retinal pigment epithelium: prevalence and ocular features in the optometric population. Ophthalmic Physiol Opt 27(6): 547-555.

2. Cohen SY, Quentel G, Guiberteau B, Coscas GJ (1993) Retinal vascular changes in congenital hypertrophy of the retinal pigment epithelium Ophthalmology 100(4): 471-474.

\section{ISSN: 2574-1241}

DOI: 10.26717.BJSTR.2019.13.002477

Martina M, Giulia M. Biomed J Sci \& Tech Res

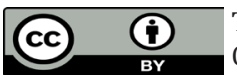

This work is licensed under Creative Commons Attribution 4.0 License

Submission Link: https://biomedres.us/submit-manuscript.php
3. Almeida A, Kaliki S, Shields CL (2013) Autofluorescence of intraocular tumours. Curr Opin Ophthalmol. 24(3): 222-232.

4. Olsen TW, Frayer WC, Myers FL, Davis MD, Albert DM (1999) Idiopathic reactive hyperplasia of the retinal pigment epithelium. Arch Ophthalmol 117: $50-54$

5. Shields JA, Shields CL, Gündüz K, Eagle RC (1999) Neoplasms of the retinal pigment epithelium. Arch Ophthalmol 117(5): 601-608.

6. Shields JA, Shields CL (2017) Tumors and Related Lesions of the Pigmented Epithelium. APJO 6 (2): 215-223.

7. Shields CL, Mashayekhi A, Ho T, Cater J, Shields JA (2003) Solitary congenital hypertrophy of the retinal pigment epithelium: clinical features and frequency of enlargement in 330 patients. Ophthalmology 110(10): 1968-1976.

8. Shields CL, Thangappan A, Hartzell K, Valente P, Pirondini C, et al. (2008) Combined hamartoma of the retina and retinal pigment epithelium in 77 consecutive patients: visual outcome based on macular versus extramacular tumor location. Ophthalmology 115(12): 2246-2252.

9. Gass JDM (1974) Choroidal nevi or benign melanomas. In: Gass JDM (Eds.), Differential diagnosis of intraocular tumors. A stereoscopic presentation. Mosby, St. Louis, USA, p. 14.

10. Pro M, Shields JA, Tomer TL (1978) Serous detachment of the macula associated with presumed choroidal nevi. Arch Ophthalmol 96(8): 1374-1377.

11. Gonder JR, Augsburger JJ, Mc Carthy EF, Shields JA (1982) Visual loss associated with choroidal nevi. Ophthalmology 89(8): 961-965.

12. Callanan DG, Lewis ML, Byrne SF, Gass JD (1993) Choroidal neovascularization associated with choroidal nevi. Arch Ophthalmol 111(6): 789-794.

13. Chien JL, Sioufi K, Surakiatchanukul T, Shields JA, Shields CL (2017) Choroidal nevus: a review of prevalence, features, genetics, risks, and outcomes. Curr Opin Ophthalmol 28(3): 228-237.

14. Shields JA, Demirci H, Mashayekhi A, Eagle RC, Shields CL (2006) Melanocytoma of the optic disk: a review. Surv Ophthalmol 51(2): 93104.

15. Kaliki S, Shields CL (2017) Uveal melanoma: relatively rare but deadly cancer. Eye 31(2): 241-257.

16. Shields CL, Furuta M, Berman EL, Zahler JD, Hoberman DM, et al (2009) Choroidal nevus transformation into melanoma: analysis of 2514 consecutive cases. Arch Ophthalmol 127(8): 981-987.

17. Almeida A, Kaliki S, Shields CL (2013) Autofluorescence of intraocular tumors. Curr Opin Ophthalmol 24(3): 222-232.

18. Shields CL, Manalac J, Das C, Ferguson K, Shields JA (2014) Choroidal melanoma: clinical features, classification and top 10 pseudomelanomas. Curr Opin Ophthalmol 25(3): 177-185.

19. Shields CL, Salazar P, Mashayekhi A, Shields JA (2009) Peripheral exudative hemorrhagic chorioretinopathy (PEHCR) simulating choroidal melanoma in 173 eyes. Ophthalmology 116(3): 529-535.

20. Shields JA, Mashayekhi A, Ra S, Shields CL (2005) Pseudomelanomas of the posterior uveal tract. Retina 25(6): 767-771.

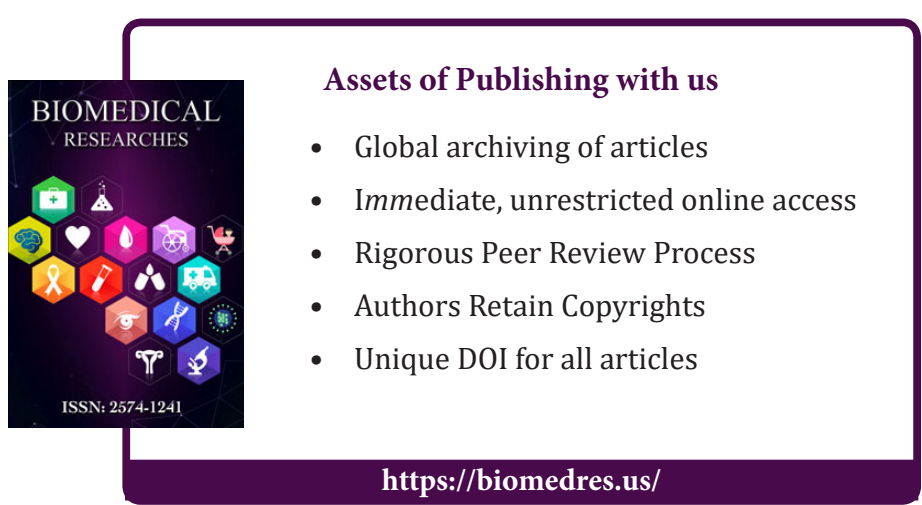

